\title{
Viability of Image Compression in Vibrothermography
}

\author{
X. Chi ${ }^{1}$ (D) $\cdot$ Y. Zhang ${ }^{1} \cdot$ D. Di Maio ${ }^{2,3} \cdot$ N.A.J. Lieven ${ }^{1}$
}

Received: 27 November 2019 / Accepted: 4 August 2020 / Published online: 12 August 2020

(C) The Author(s) 2020

\begin{abstract}
This research focuses on the verification of the viability of image compression in infrared thermography in order to address the problem of data storage. Specifically, images from vibrothermographic tests were utilized due to their special characteristics compared to the results from alternative infrared thermography techniques, which are able to introduce additional uncertainties to the compression process. In this research, an adaptive algorithm based on the lifting discrete wavelet transform and setpartitioning embedded blocks was used for image compression. Five different methods, namely the compression ratio, mean squared error, peak signal-to-noise ratio, structural similarity index and coordinate modal assurance criterion, were applied to evaluate the performance of the compression process while identifying and locating the regions affected more significantly after image compression. Feature extraction through the independent component analysis was then applied to the images to separate the features such as the hot spots so that the influence from the image compression process on each important feature could be evaluated independently. In this article, the theoretical background of the applied data processing techniques is firstly presented. Through two sets of data acquired from vibrothermographic tests on an aerospace-grade composite plate containing delamination, the effects of the image compression process on the relevant hot spots are evaluated, and the effectiveness of the compression process is verified. It is demonstrated that the compression process was able to reduce the size of the images significantly without adversely affecting the quality of the important features indicating the presence of damage. The major characteristics of the key features have been successfully preserved after effective image compression.
\end{abstract}

Keywords Structural health monitoring $\cdot$ Infrared thermography $\cdot$ Vibrothermography $\cdot$ Image compression $\cdot$ Feature extraction * Composite material

\section{Introduction}

In the area of non-destructive testing and evaluation (NDT\&E), infrared thermography (IRT) has evolved rapidly during the past decades thanks to the advances in infrared (IR) cameras and lenses. Among the industrially utilized IRT techniques, vibrothermography is a special application which usually relies on the friction inside the inspected structure as the heat source. In a vibrothermographic test, the inspected structure is excited using

X. Chi

xintian.chi@bristol.ac.uk

1 Department of Aerospace Engineering, University of Bristol, Bristol, England, UK

2 Department of Mechanical Engineering, University of Twente, Enschede, Netherlands

3 Department of Mechanical Engineering, University of Bristol, Bristol, England, UK mechanical forces or acoustic/ultrasonic waves at a single frequency — or multiple frequencies - which induce structural vibration. During the vibration, friction is often generated within the structure due to relative motion between surfaces and components. Because of the friction-based heat generation mechanism, vibrothermography is known for its effectiveness in detecting cracks and delamination [1]. Another operational advantage of vibrothermography is that as heat is generated within the structure, the travel distance of the thermal wave is shortened compared to the case in conventional active IRT tests where heat flows are applied externally to the material surface.

Compared to other commonly deployed NDT\&E techniques, IRT approaches, including vibrothermography, have distinctive advantages in operating conditions due to the capability to perform rapid non-contact scans of large areas with short measurement time. The results from IRT tests are usually presented in the form of high-definition two-dimensional images or videos generated from the temperature data acquired by IR cameras. These high-definition images or videos 
are able to provide clear and robust demonstrations of the condition of the inspected structure, in which the location of the damage and defects can be revealed by the hot spots and/or cold spots. Due to the uncomplicated demonstration of measurement results, these images are also relatively easy to analyze. These advantages make IRT attractive in structural health monitoring and damage detection, especially when applied to large engineering structures where the instrumentation and measurement time required for other non-contact methods is usually considerably longer while the contact sensingsuch as accelerometers and strain gauges - would miss large areas of the structure.

However, these easy-to-process high-definition images and videos come at a price. The requirements on storage space are one of the unavoidable problems of IRT, which is more significant when the safety requirement is critical so that online condition monitoring must be applied. In this case, a considerable amount of data needs to be stored to allow data traceability. Additionally, the demands on storage space have been increasing rapidly due to continuously advancing IR cameras, which are able to record data with higher resolution and frame rates.

In order to address this issue, data processing techniques such as feature extraction and data compression can be utilized.

There have been research outputs showing the possibility of using feature extraction on the thermal images acquired from IRT tests so that the images can be decomposed to underlying components containing different features [2] [3]. Through feature extraction, the components containing meaningful features such as the hot spots in the damaged regions can be extracted and preserved while the other components can be discarded in order to reduce the requirements on data storage [4].

However, the feature extraction process eliminates the temporal sequence of the data in exchange for the individual features, while in fact, the extracted results are not the identical representations of the original data at any specific time. For this reason, if the data at any specific time, or the time itself, is important, such as the time at which a critical event happens, the inclusion of feature extraction is not preferable.

A practical solution is to compress the raw data captured by IR cameras directly, which lowers the requirements for data storage from the outset while still maintaining the correct sequence of the data.

For the purpose of damage detection, Lugin et al. [5] developed a compression algorithm for data acquired from pulsed thermography tests, which was able to achieve a compression factor of up to 24-55 at a reproduction accuracy. Using medical infrared images as the vehicle, Schaefer et al. [6] compared several compression techniques, such as the Lossless JPEG, JPEG-LS, JPEG2000, PNG, and CALIC and demonstrated the possibility of compressing infrared images without losing critical information in medical applications.
However, research outputs demonstrating the viability of applying image compression to results from vibrothermographic tests are still limited. In vibrothermography, the results differ from the images captured in alternative IRT approaches in terms of several factors, which can add extra uncertainties to the compression process.

Firstly, during vibrothermographic tests, as external excitation is applied to the structure to induce vibration, the inspected object is not static during the tests. This phenomenon is particularly noticeable for vibration with high amplitude so that the motion of the inspected object can be reflected in the images acquired from vibrothermographic tests.

Secondly, in vibrothermographic tests, multiple hot spots may be generated, each from a different physical source. As a matter of fact, friction is not the only source of heat generation during a vibrothermographic test. There is also heat generated through other mechanisms such as plasticity-induced heat generation, viscoelastic heating and thermoelastic effect, although the frictional heat generation is often the most dominant source of heat generation in vibrothermographic tests [1] [7], which includes the experimental tests carried out in this research. However, even in situations where only frictional heat generation is considered, multiple hot spots always appear at different locations and different times. Among these hot spots, some are caused by the damage and defects while others may be caused by irrelevant features such as the less important interfaces. Typically, each hot spot often has a unique shape and amplitude. Specifically, damage and defects in regions with greater strain energy are more likely to develop higher frictional heat generation as the amount of heat generated through friction is determined by frictional force and slip rate (speed of relative motion) between the contact surfaces [1], which are positively correlated with local strain energy [3] [8]. The existence of multiple hot spots with varying amplitude across different frames of images is expected to present more challenges to the compression process.

Regarding the issue of additional irrelevant hot spots, a possible solution is to perform more customized excitation in order to induce the resonance of the damage and defects if there is sufficient knowledge of the damage and defects, which might provide more localized heat generation that can eliminate or reduce the magnitude of other hot spots experimentally. However, the prior knowledge of the characteristics of the potential damage and defects is not always available, in which case this approach is compromised.

Additionally, hot spots are likely to exhibit temperature fluctuations during measurement because of the inconstant heat generation rates during vibration caused by varying excitation force and other factors. Random fluctuations can also appear due to reasons such as camera noise, uneven emissivity and viewing angle of the IR camera.

Moreover, for in-field applications, reflections - including static and moving ones - may become unavoidable, which 
add extra noise to the measured results. Although image or pixel subtraction methods are often utilized to reduce reflections, removing reflections completely is still difficult. For this reason, in one of the experimental tests carried out in this research, it was not attempted to control the reflections in order to add extra challenges artificially, which should benefit the reliability and applicability of the conclusions obtained from this research.

Considering that data storage is an unavoidable problem of IRT in both laboratory tests and industrial applications, verifying the viability of applying image compression to results from vibrothermography, or other IRT approaches, appears to be necessary for improving its practicality in relevant applications.

In this research, data presented in the form of thermal images were acquired from two vibrothermographic tests on an aerospace-grade composite plate containing delamination. During the vibrothermographic tests, multiple hot spots generated through different mechanisms were developed. Specifically, in the second test, reflections were not eliminated which added extra noise to the measured results. After the experimental tests, the thermal images were firstly compressed to four different levels using an adaptive compression algorithm based on lifting discrete wavelet transform and setpartitioning embedded blocks [9] [10]. Five different methods, namely the compression ratio, mean squared error, peak signal-to-noise ratio, structural similarity index and coordinate modal assurance criterion, were applied to evaluate the performance of the compression process while identifying and locating the regions affected more significantly after image compression. Next, each set of data was processed through feature extraction, which separated the different hot spots so that the effects of the compression process on each individual hot spot could be assessed independently. The original images and the compressed data, as well as their respective extracted features, were compared to enable the consideration of the influence of the image compression process on the important features in the thermal images, such as the hot spots indicating the presence of damage, so that the viability of image compression in IRT, particularly vibrothermography, could be demonstrated.

\section{Algorithm Overview and Theoretical Background}

As explained in the previous section, the two main constituent components for data processing in this research are image compression and feature extraction.

In this research, the image compression process was achieved by an adaptive compression scheme [9] [10]. The compression algorithm is composed of a lifting discrete wavelet transform (LDWT) with set-partitioning embedded blocks
(SPECK) that efficiently orders the wavelet coefficients by significance. The algorithm exploits the clustering of energy found in the transformed domain and concentrated on those areas of the set which have high energy. This allows those signals with higher information to be encoded first based on their energy content. The output of the SPECK module is the input to an optimized context-based arithmetic coder that generates the compressed bitstream.

Specifically, the advantages of the LDWT scheme are:

1. Lifting allows for adaptive wavelet transform;

2. The LDWT allows lossless reversible integer-to-integer transform; and

3. Lifting allows fast forward and inverse wavelet transforms and simple implementation.

The lifting scheme enables an adaptive wavelet transform, which means that the transform can start the analysis of a function from the most important (fundamental) layers and then build the detail layers by refining only the areas of interest. In the decoding part, the decoder can adaptively refine the detail information based on the requirements. An efficient wavelet coefficient sorting algorithm such as SPECK is helpful to sort subsets of wavelet coefficients by significance.

The SPECK uses three dynamic lists to store significant information of wavelet coefficients for compression purposes, which are the list of significant pixels (LSP), list of insignificant pixels (LIP) and list of insignificant sets (LIS). To check the significance, the algorithm compares the wavelet coefficients in a set with respect to a sequence of decreasing threshold. In case that the wavelet coefficient has its magnitude above the threshold, the set is split into four subsets whose significance with respect to the threshold is tested, otherwise, the set is not split. Finally, the algorithm rescans those pixels found to be significant with respect to previous thresholds and refines their magnitude. The function used for testing the set significant state is defined by the following formula:

$S_{i}(\Gamma)=\left\{\begin{array}{c}1, \quad 2^{i} \leq \max _{(m, n) \in \Gamma}\left\{\left|c_{m, n}\right|\right\} \leq 2^{i+1} \\ 0, \text { otherwise }\end{array}\right.$

and

$i=\left\lfloor\log _{2}\left(\max _{(m, n)}\left\{\left|c_{m, n}\right|\right\}\right)\right\rfloor$,

where $c_{m, n}$ is the coefficient value for position $(m, n)$ in the wavelet sub-bands. The $\Gamma$ indicates the set of coefficients and $S_{i}(\Gamma)$ is used for the significant state of the set $\Gamma$, where 1 indicates the set is significant to the threshold $T=2^{i}$, and 0 indicates it is insignificant and a bit will be emitted for the 
entire insignificant set. The insignificant pixels and insignificant sets are put in LIP and LIS, respectively; the significant pixels are put in the LSP.

The context modelling in SPECK has been achieved by arithmetic coding (AC), which can obtain optimal performance thanks to its ability to represent information with fractional bits. AC has been widely utilized by various image and video codecs, such as the QM coder in JPEG [11], the MQ coder in JPEG2000 [12], and the context-based adaptive binary arithmetic coder (CABAC) in H.264/AVC and HEVC [13]. The context modelling provides estimates of the conditional probabilities of the coding coefficients and exploits the redundancy present in the neighborhood of the current coefficient to encode.

The arithmetic coder employed in this work is the MQcoder as specified in the JBIG and JPEG2000 standard [12] [14]. The MQ-coder is a binary alphabet adaptive multiplication-free arithmetic coder. A probability model has been implemented as a 47-state Finite State Machine (FSM) for the coding process. In the FSM, each state contains coding information, a set of probability mapping rules (look-up table) which are used to interpret and manipulate the context state associated with the current coding context [14]. This approach can estimate the probability of the context more efficiently and take into consideration the non-stationary characteristic of the symbol string. The MQ-coder can adapt to the input bitstream by estimating the probability through the use of two tables: Dynamic table and Static table. The Dynamic table contains the information of each context, and the Static table describes the probability state transition for MQ-coder [14].

In this research, the experimental results indicated that the data compressor was able to efficiently compress the signals from the vibrothermographic tests with high-quality reconstruction as it will be shown later in this article. The compression ratio (CR) was utilized to measure the relative reduction in the size of data after the image compression process, which can be easily calculated as follows:

$\mathrm{CR}=\frac{L_{\text {orig. }}}{L_{\text {comp. }}}$

where $L_{\text {orig. }}$ and $L_{\text {comp. }}$ are the sizes of the original data and the compressed output respectively.

In addition to the overall compression ratio, for the purpose of damage detection and structural health monitoring, it must be verified that the regions that contained valuable information and meaningful features such as the hot spots were less affected during the compression process as the compression mostly influenced the areas without meaningful features. In order to achieve this objective, after the image compression process, three different methods were utilized to identify and locate the differences between the original images and the compressed data. Specifically, these three techniques are the mean squared error (MSE), structural similarity (SSIM) index and coordinate modal assurance criterion (COMAC).

Among these three methods, the MSE provides the simplest approach. Firstly, the MSE between two images $I$ and $K$ which have the same pixel resolution $M \times N$ can be calculated from:

$\mathrm{MSE}=\frac{1}{M N} \sum_{m=1}^{M} \sum_{n=1}^{N}[I(m, n)-K(m, n)]^{2}$

where $I(m, n)$ is the intensity of the pixel $(m, n)$ in the first image while $K(m, n)$ is the intensity of the same pixel in the second image.

Now if $I$ represents a set of original images that have not been compressed while $K$ represents the corresponding set of images after image compression, the MSE for each pixel between every image pair can be calculated using:

$\operatorname{MSE}\left(m_{i}, n_{i}\right)=\left(I\left(m_{i}, n_{i}\right)-K\left(m_{i}, n_{i}\right)\right)^{2}$,

where, for the specific application in this research, $I\left(m_{i}, n_{i}\right)$ is the intensity of the pixel $(m, n)$ in the $i^{t h}$ image of the original data set and $K\left(m_{i}, n_{i}\right)$ is the intensity of the same pixel in the corresponding image of the compressed data set. After calculating the MSE for every image pair, the average MSE for the pixel $(m, n)$ between the two sets of images can be calculated as follows:

$\operatorname{MSE}_{\text {avg. }}(m, n)=\frac{1}{J} \sum_{i=1}^{J} \operatorname{MSE}\left(m_{i}, n_{i}\right)$.

where it has been assumed that each set contains $J$ images. The calculated average MSE map can then be utilized to visualize the pixel-wise differences between the two sets of images. Eventually, a single MSE value can be calculated to evaluate the overall difference between the two sets of images using:

$\mathrm{MSE}=\frac{1}{M N} \sum_{m=1}^{M} \sum_{n=1}^{N} \operatorname{MSE}_{\text {avg. }}(m, n)$

Based on the overall MSE, the peak signal-to-noise ratio (PSNR) can be calculated between the original and the compressed data from:

$\operatorname{PSNR}=10 \log _{10}\left(\frac{\mathrm{MAX}_{I}^{2}}{\mathrm{MSE}}\right)$

where MSE is the overall mean squared error between the original images $I$ and the compressed images $K$. MAX $I$ is the maximum possible pixel value of the image, which is 255 in this case. 
Compared to the simple and straightforward methods such as the MSE and PSNR, the SSIM was designed as an improvement by considering three components, which are the luminance $l(x, y)$, the contrast $c(x, y)$ and the structure $s(x, y)$ [15], so that:

$l(x, y)=\frac{2 \mu_{x} \mu_{y}+C_{1}}{\mu_{x}^{2}+\mu_{y}^{2}+C_{1}}$,

$c(x, y)=\frac{2 \sigma_{x} \sigma_{y}+C_{2}}{\sigma_{x}^{2}+\sigma_{y}^{2}+C_{2}}$

and

$s(x, y)=\frac{\sigma_{x y}+C_{3}}{\sigma_{x} \sigma_{y}+C_{3}}$,

where $\mu_{x}$ is the mean intensity of a window $x, \mu_{y}$ is the mean intensity of another window $y, \sigma_{x}^{2}$ is the variance of $x$, $\sigma_{y}^{2}$ is the variance of $y$ and $\sigma_{x y}$ is the covariance of $x$ and $y$. The constants $C_{1}, C_{2}$ and $C_{3}$ are included to avoid instability when the denominators are close to zero [15]. The constants can be calculated using:

$C_{1}=\left(K_{1} L\right)^{2}$

$C_{2}=\left(K_{2} L\right)^{2}$

and

$C_{3}=\left(K_{3} L\right)^{2}$,

where $L$ is the dynamic range of the pixel values while $K_{1}$, $K_{2}$ and $K_{3}$ are arbitrary small constants that are much less than $1[15]$.

By combining the three components, the SSIM index can be calculated as follows:

$\operatorname{SSIM}(x, y)=[l(x, y)]^{\alpha} \cdot[c(x, y)]^{\beta} \cdot[s(x, y)]^{\gamma}$.

By setting $\alpha=\beta=\gamma=1$ and $C_{3}=C_{2} / 2$, it can be simplified to:

$\operatorname{SSIM}(x, y)=\frac{\left(2 \mu_{x} \mu_{y}+C_{1}\right)\left(2 \sigma_{x y}+C_{2}\right)}{\left(\mu_{x}^{2}+\mu_{y}^{2}+C_{1}\right)\left(\sigma_{x}^{2}+\sigma_{y}^{2}+C_{2}\right)}$.
In this research, $x$ was taken from an image of the original data set while $y$ was the counterpart from the corresponding image of the compressed data set. After calculating the local SSIM values between every image pair, the average local SSIM for a window $m$ between the two sets of images can be obtained from:

$\operatorname{SSIM}_{\text {avg. }}\left(x_{m}, y_{m}\right)=\sum_{i=1}^{J} \operatorname{SSIM}\left(x_{m i}, y_{m i}\right)$.

where it has been assumed that each set contains $J$ images.

Similar to the MSE, a single SSIM value can be calculated to represent the overall similarity between two images $I$ and $K$. This was defined as the mean SSIM (MSSIM) [15]. By assuming that there are $M$ windows in each image, it can be calculated from:

$\operatorname{MSSIM}(I, K)=\frac{1}{M} \sum_{m=1}^{M} \operatorname{SSIM}\left(x_{m}, y_{m}\right)$.

This can then be extended to two sets of images by substituting the average local SSIM between the two sets of images, which is the SSIM $_{\text {avg. }}$ from Eq. 17, for the SSIM in Eq. 18, so that a single SSIM value between the two sets of images can be calculated. Additionally, the results calculated from Eq. 17 can be utilized to construct a pixel-by-pixel map visualizing the influence from the image compression process, where the details can be found in the article by Wang et al. [15]. In the SSIM map, regions with smaller local SSIM values correspond to areas that have been affected more significantly through the image compression process.

Eventually, the coordinate modal assurance criterion (COMAC), originally developed to identify the degrees-offreedom that cause differences between two sets of mode shapes [16], was utilized as the third method to locate the differences between the original and the compressed images. The COMAC can be calculated from:

$\operatorname{COMAC}(m)=\frac{\left(\sum_{i=1}^{J}\left|\left(\varphi_{A}\right)_{m i}\left(\varphi_{X}\right)_{m i}^{*}\right|\right)^{2}}{\sum_{i=1}^{J}\left(\varphi_{A}\right)_{m i}^{2} \sum_{i=1}^{J}\left(\varphi_{X}\right)_{m i}^{* 2}}$,

where, for the specific application in this research, $\left(\varphi_{A}\right)_{m i}$ represents the intensity of the $m^{\text {th }}$ pixel in the $i^{\text {th }}$ image of the first data set. $\left(\varphi_{X}\right)_{m i}$ is the intensity of the same pixel in the corresponding image in the second data set and $J$ is the total number of correlated image pairs. The COMAC, which is a vector, can be converted back to a matrix which has the same dimension as the pixel resolution of the original images and every element in COMAC can be placed back at their original pixel position. The value of each element in the COMAC vector can be used to define the height and/or the color of 
Fig. 1 The rig for the experimental tests

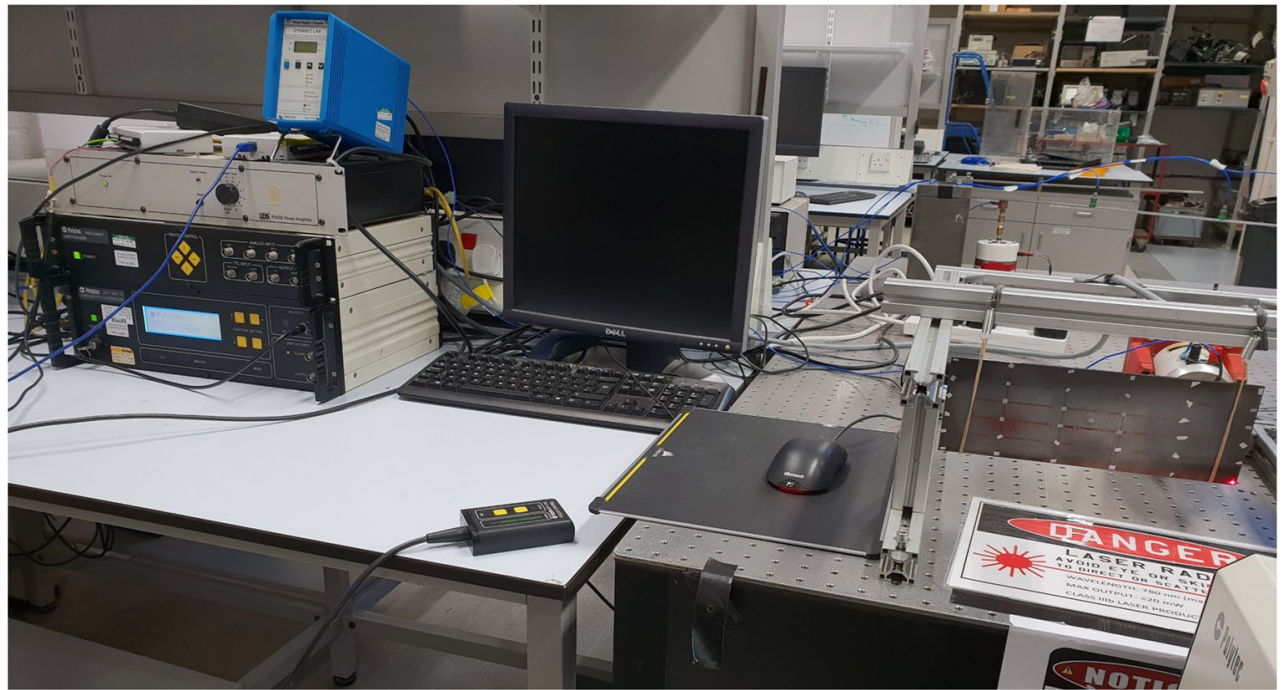

each pixel in the re-created image so that the pixels causing the differences between two sets of images can be located easily. The COMAC is normalized so always has a value between 0 and 1. A larger COMAC value indicates higher similarities at a specific element, which is a pixel in this case, between the two sets of data.

In this research, the MSE, SSIM and COMAC maps were calculated between the original and the compressed sets of data. The regions affected more significantly in the image compression process would have higher MSE values but lower SSIM and COMAC values than those affected less. Based on this information, the areas influenced more significantly through the image compression process could be identified and located clearly, with which the effectiveness of the image compression could be appraised.

To complete the process, the original and the compressed data sets were further processed through feature extraction. Feature extraction was performed after the completion of image compression, which attempted to separate the underlying features in the thermal images such as the hot spots. With the different features separated, the effects of the compression process on each individual hot spot could be assessed independently.

In feature extraction, it is assumed that each underlying feature is generated by a unique physical source. For the case in this research, these unique features were mixed to form the thermal images that were captured by the IR camera. The mixing process can be described as:

$X=A S$,

where $X$ is the observed mixtures, which were the thermal images in this research, $S$ is the source signals, which were the underlying features such as the hot spots generated by different physical sources, and $A$ is the matrix that performs the mixing process.

The feature extraction can be considered as a reverse of the mixing process, so that:

$Y=W X$

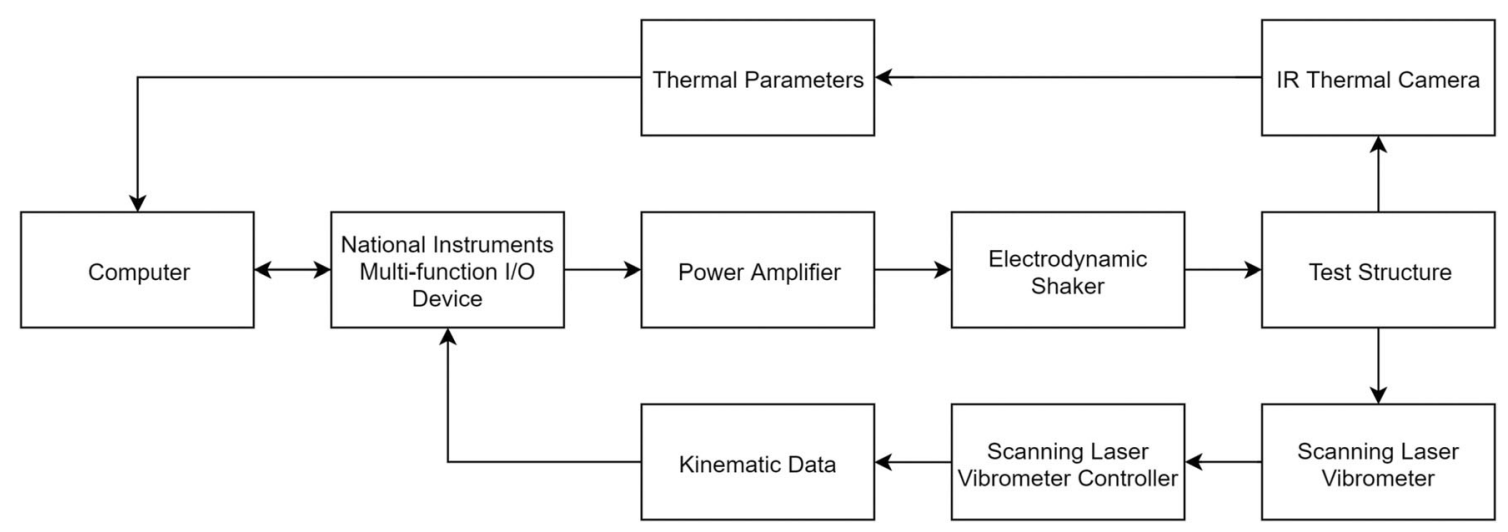

Fig. 2 The flowchart of the experimental tests 
Fig. 3 a The suspended plate and b the attachment point of the electrodynamic shaker

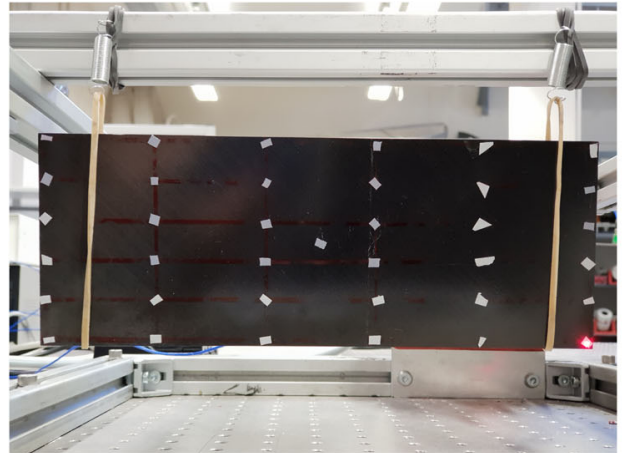

a

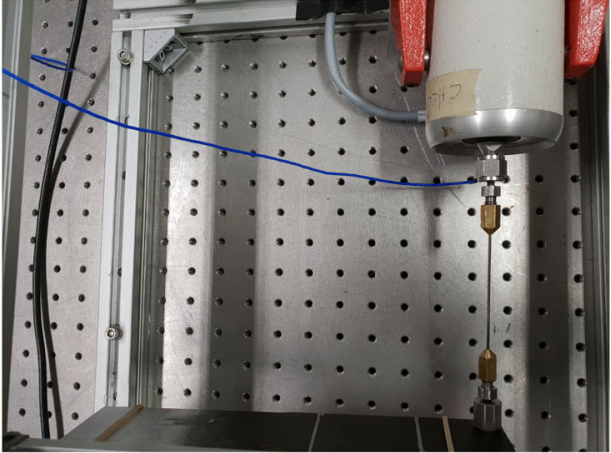

b where $Y$ contains the separated features. The objective of a feature extraction process is to calculate a proper $W$ so that the extracted signals $Y$ are as close to the original signals $S$ as possible. These components in $Y$ are assumed to be mutually uncorrelated (as in the principal component analysis (PCA) and factor analysis (FA)) [4] or statistically independent (as in the independent component analysis (ICA)) [17]. $W$ is the matrix that performs the unmixing process, which needs to be determined in order to calculate the correct components from the feature extraction.

Multiple feature extraction algorithms can achieve this objective of calculating the unmixing matrix $W$ or its constituting weight vectors $w_{i}$, among which PCA, FA and ICA are three of the most common techniques [17]. Among these three techniques, ICA, specifically, FastICA [18], was used in this research, as it has been verified on the thermal images used in this research that it was able to separate the underlying features successfully while still maintaining their correct characteristics, which was unable to be achieved by PCA [3].

However, despite being able to provide more robust results than alternative techniques in most situations, FastICA has certain limitations that must be considered.
Firstly, due to the calculation algorithm of FastICA, the number of extracted components is equal to the number of input signals provided, given that no dimensionality reduction is performed. In most cases, the number of extracted components cannot reflect the actual number of underlying features.

Another limitation of FastICA that is relevant to IRT is that FastICA cannot determine the sign or scale of each component, which means the heat generated by some heat sources may appear as cold spots in the components extracted by FastICA due to the change in sign [19]. For this reason, the extracted components in this research were further processed so that the hot spots always appear as red in the generated images of the extracted components. Another consequence of this limitation is that the values of the elements in the components extracted by FastICA are not equivalent to the original temperature data due to the change in scale, although the scale does not affect the relative magnitude of data across pixels in each extracted component.

Another characteristic of FastICA to be considered is that the order of the extracted components is not determined by any meaningful criterion, although this can be solved by introducing additional calculations, such as using kurtosis, to re-
Fig. 4 Strain energy distribution of the first mode of vibration

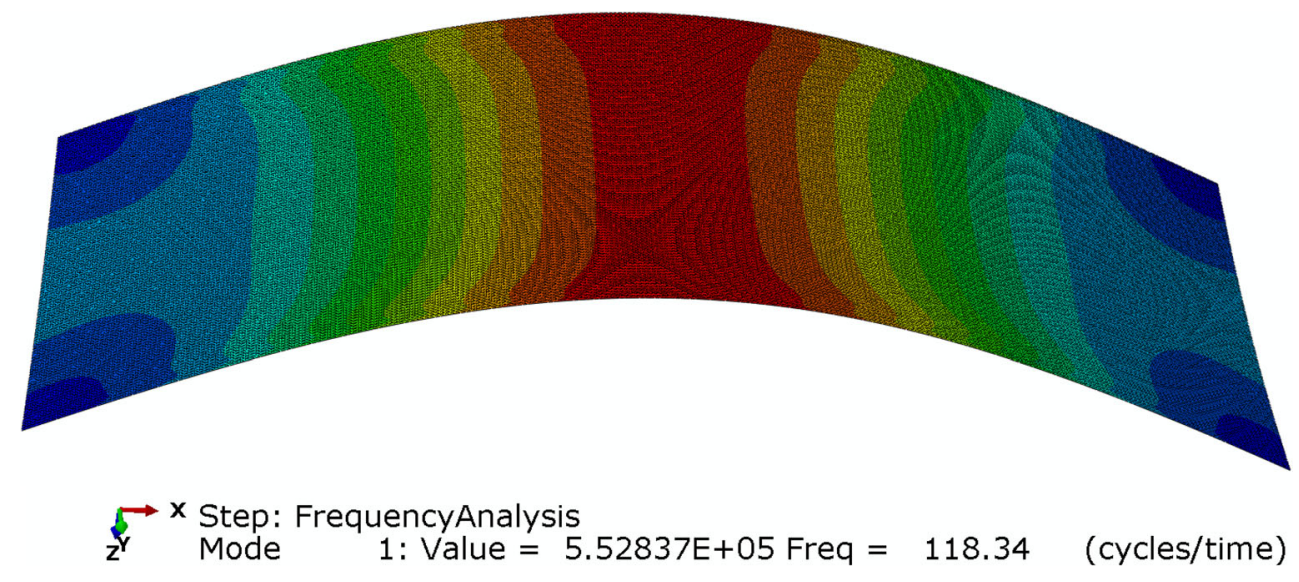


Table 1 Timeline of the first vibrothermographic test

\begin{tabular}{ll}
\hline Time (min:s) & Event \\
\hline 0:30 & The first photograph below (Fig. 5 (a)) was taken. \\
5:00 & The electrodynamic shaker was turned on. \\
5:30 & The second photograph below (Fig. 5 (b)) was taken. \\
25:00 & The electrodynamic shaker was turned off. \\
25:15 & The third photograph below (Fig. 5 (c)) was taken. \\
45:00 & The fourth photograph below (Fig. 5 (d)) was taken. \\
\hline
\end{tabular}

order the extracted components, which has been demonstrated by the authors [3].

\section{Experimental Development and Data Acquisition}

As explained in the section "Introduction", the experimental data were acquired from two vibrothermographic tests performed on an aerospace-grade composite plate containing delamination. The plate was made of HexPly® 8552 AS4 epoxy matrix woven carbon prepregs composite material which is commonly utilized in primary aerospace structures [20]. The damage considered was vertical delamination created in the central region of the plate through dynamic fatigue.

Composite materials are used more and more routinely in industries due to their significant advantages in physical properties in terms of strength-to-weight ratio when compared to traditional materials such as metals. However, the unique structure of ply-based composite materials has brought in new damage mechanisms such as delamination. Delamination is arguably the most common and most challenging type of damage in composite materials used in aerospace-related applications. However, this type of damage can be difficult to detect as they are rarely observable on the surface of the material. For IRT specifically, the hot spots generated due to delamination are also more difficult for IR cameras to capture than those generated by visible defects. These reasons justify the attention that

Table 2 Timeline of the second vibrothermographic test

\begin{tabular}{ll} 
Time (min:s) & Event \\
\hline 0:30 & The first photograph below (Fig. 6 (a)) was taken. \\
5:00 & The electrodynamic shaker was turned on. \\
6:00 & The second photograph below (Fig. 6 (b)) was taken. \\
20:00 & The third photograph below (Fig. 6 (c)) was taken. \\
35:00 & The fourth photograph below (Fig. 6 (d)) was taken. \\
35:00 & The electrodynamic shaker was turned off. \\
40:00 & The fifth photograph below (Fig. 6 (e)) was taken. \\
50:00 & The sixth photograph below (Fig. 6 (f)) was taken. \\
\hline
\end{tabular}

composite materials have received in research activities involving IRT [21] [22] [23] [24] [25] [26]. Because of the properties of the structure being inspected in this research and the type of damage, vibrothermography was more suitable than the conventional heat-based IRT approaches thanks to the frictionbased heat generation mechanism.

As explained in the section "Introduction", frictional heat generation rate during vibrothermographic tests is positively correlated with local strain energy. For a vibrating structure, high strain energy is achieved around resonance, at which response of the structure is maximized. For this reason, the calculation of strain energy distribution in the structure can be achieved by a modal analysis.

Specifically, modal analysis is able to numerically calculate and experimentally measure the natural frequencies (which are approximately equal to the resonant frequencies for structures with relatively low damping) as well as the mode shape and the associated strain energy distribution of each mode of vibration. When a structure is in an operating condition, its deflection shape is a combination of all excited mode shapes. For this reason, calculating the strain energy map of each mode of vibration is able to predict the actual strain energy distribution in the structure [27].

For active vibrothermographic testing where external excitation is applied to the structure to induce vibration, with the necessary knowledge of the distribution of strain energy at each resonance, parameters of the excitation force can be selected to target a specific mode of vibration in order to ensure high strain energy concentration in the interested regions during the structure's vibration.

For this reason, in this research, in order to perform the vibrothermographic test effectively and efficiently, a modal test was performed first to calculate the modal parameters of the structure so that the proper excitation frequency and location could be selected. The rig set-up and the flowchart demonstrating the procedures of the experimental tests are shown in Figs. 1 and 2. During all tests, the composite plate was suspended using two springs with elastic bands and connected to an LDS V201 electrodynamic shaker through a pushing rod which was screwed into a connector glued onto the back of the plate, as shown in Fig. 3. As shown in Fig. 3 (a), multiple reflective tapes were glued onto the plate to allow for the measurement using a scanning laser Doppler vibrometer during the modal test. All reflective tapes but one were then removed before the vibrothermographic tests.

After the completion of the modal analysis, the first mode of vibration was selected for the vibrothermographic test. The highdefinition strain energy distribution in the structure during this mode of vibration was calculated from an updated finite element model. The strain energy distribution was superimposed on its corresponding mode shape and shown in Fig. 4.

As shown in Fig. 4, the central region of the plate where the damage was located had high strain energy concentration in 


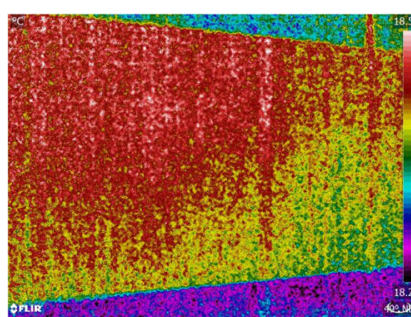

a

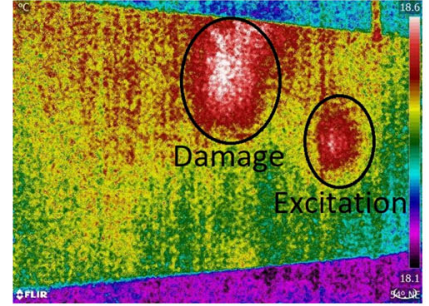

b

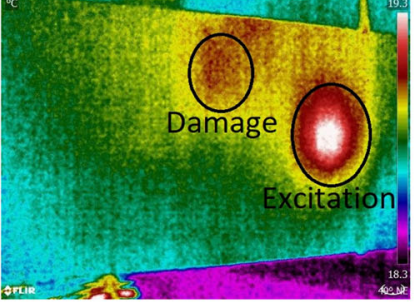

c

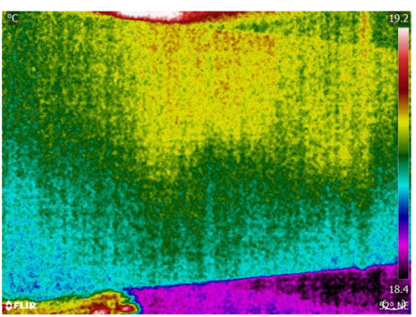

d

Fig. 5 Photographs taken at (a) 0:30, (b) 5:30, (c) 25:15 and (d) 45:00 of the first vibrothermographic test

the first mode of vibration, which demonstrated its suitability for vibrothermographic testing.

During the vibrothermographic test, the electrodynamic shaker was set to provide a sinusoidal excitation force with low amplitude. The excitation frequency was set to match the natural frequency of the first mode of vibration of the composite plate, which was experimentally measured as $119 \mathrm{~Hz}$. The location of excitation was also adjusted in the vibrothermographic tests due to the limitations from the aspect ratio of the IR camera. As mentioned previously, all reflective tapes except for the one at the excitation point were removed for the vibrothermographic tests. The remaining reflective tape was left to enable the measurement of the driving point frequency response function which was used to ensure that the natural frequencies were not noticeably altered after changing the location of the excitation point.

A FLIR T650sc IR camera was used in the vibrothermographic tests for data acquisition. The camera has resolution of $640 \times 480$ and thermal sensitivity of $0.02{ }^{\circ} \mathrm{C}$, which means it was able to capture the subtle temperature changes in the inspected structure during the vibrothermographic tests.

As mentioned previously, two experimental vibrothermographic tests were performed. In the first test, barriers were used to block the light to control the reflections on the composite plate, while in the second test the IR camera was placed directly in front of the plate, in which case the reflections were significantly stronger.

In both tests, data recording had been started before the shaker was turned on in order to capture the initial temperature of the plate. After five minutes, the electrodynamic shaker was turned on to provide the excitation force. Due to the frequency and location selected for the excitation force, the deflection shape of the plate closely resembled the mode shape of its first mode of vibration. During the test, heat was generated in the damaged region because of the friction caused by the vibration. The frictional heat generation was able to be captured by the IR camera so that a hot spot could be observed in the damaged region. A separate hot spot was also developed at the excitation point primarily due to the heat transferred from the shaker into the plate through the pushing rod. At the end of the active testing, the shaker was turned off, so the temperature of the plate started to decrease, during which the heat concentration at the excitation point and in the damaged region gradually dissipated. In both tests, data were recorded at 15 -s intervals. The first test lasted $45 \mathrm{~min}$, during which 181 frames of data were recorded. The

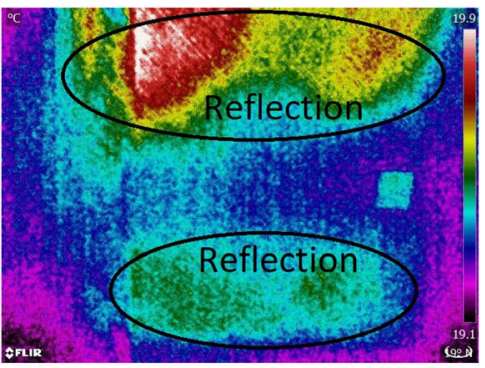

a

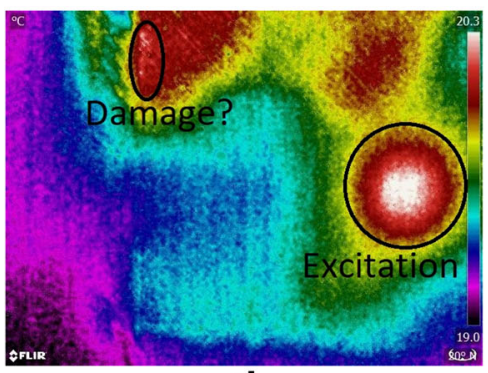

d

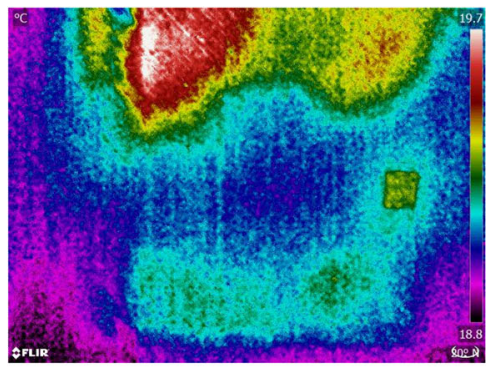

b

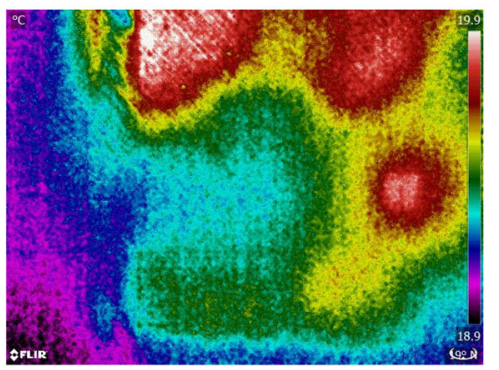

e

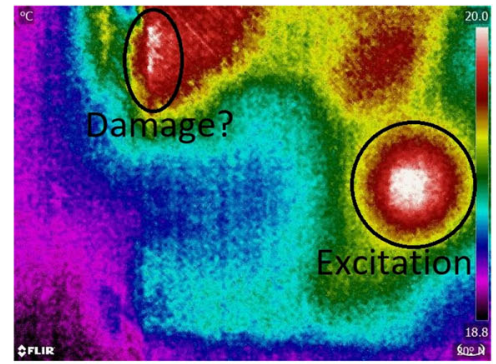

C

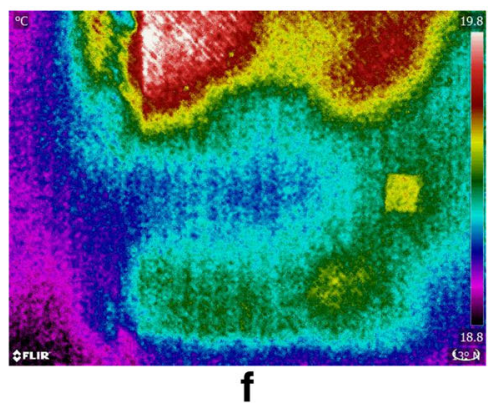

Fig. 6 Photographs taken at (a) 0:30, (b) 6:00, (c) 20:00, (d) 35:00, (e) 40:00 and (f) 50:00 of the second vibrothermographic test 
Table 3 Compression results, the first vibrothermographic test

\begin{tabular}{lllll}
\hline & $\mathrm{C} 0$ & $\mathrm{C} 1$ & $\mathrm{C} 2$ & $\mathrm{C} 3$ \\
\hline Resolution & $640 \times 480$ & & & \\
Number of Frames & 181 & & & \\
Original Size & $164,306 \mathrm{~KB}$ & & & \\
CR & 14.13 & 30.55 & 76.00 & 184.41 \\
MSE & 13.8974 & 27.1917 & 47.5943 & 65.3152 \\
PSNR & $36.7015 \mathrm{~dB}$ & $33.7864 \mathrm{~dB}$ & $31.3553 \mathrm{~dB}$ & $29.9807 \mathrm{~dB}$ \\
SSIM & 0.9101 & 0.8456 & 0.7727 & 0.7293 \\
Avg. COMAC & 0.9993 & 0.9986 & 0.9974 & 0.9963 \\
Size (Change) & $11,627 \mathrm{~KB}(-92.92 \%)$ & $5378 \mathrm{~KB}(-96.73 \%)$ & $2162 \mathrm{~KB}(-98.68 \%)$ & $891 \mathrm{~KB}(-99.46 \%)$ \\
\hline
\end{tabular}

second test lasted $50 \mathrm{~min}$, during which 200 frames of data were recorded. The timelines of the two vibrothermographic tests are described in Tables 1 and 2 respectively.

As shown in Fig. 5 (a), the maximum temperature variance across the plate was less than $0.2^{\circ} \mathrm{C}$ before the electrodynamic shaker was turned on. After a 30-s excitation, two separate hot spots could be observed clearly in the damaged region and at the excitation point respectively, as shown in Fig. 5 (b). Fig. 5 (c) shows that the heat concentration in the damaged region dissipated swiftly within $15 \mathrm{~s}$ after the shaker was turned off, while it took 20 min (Fig. 5 (d)) for the hot spot at the excitation point to be fully dissipated. The reason could be mostly attributed to the heat generated in the shaker, which was significantly higher than the heat generated in the plate, continued to be transferred into the plate after the shaker was turned off.

Within the captured images, it can be observed that multiple concerns regarding the results from vibrothermographic tests that would create additional uncertainties to the image compression process, which are summarized and explained in section "Introduction", have been confirmed.

Firstly, there were two main hot spots on the plate, one generated by friction and the other caused primarily by heat transfer. There were additional hot spots in the background, as shown in Fig. 5 (c) and Fig. 5 (d). The two main hot spots started to appear at different time, and the peak amplitude was also different. Specifically, the frictional heat generation started more rapidly, but the continuous heat transfer from the shaker was able to eventually accumulate a more significant amount of energy. Color anomalies and fluctuations caused by unwanted factors, such as camera noise, uneven emissivity and viewing angle, could also be observed clearly as shown in Fig. 5 (a), which was taken before the shaker was started.

In addition to the concerns mentioned above, reflections were included in the second test, where barriers were removed, and the camera was placed directly in front of the plate.

The results from the second test were considerably more complicated due to the additional reflections caused by the removal of the barrier. Specifically, the reflections were predominantly caused by the camera which was placed directly in front of the plate in this test. The reflections were captured clearly as shown in Fig. 6 (a). In the first test, it only took four seconds for a hot spot to emerge in the damaged region while in this test the heat generated in the damaged region was significantly less noticeable because it was overshadowed by the strong reflections (Fig. 6 (b)). Fig. 6 (c) and Fig. 6 (d) show that an obvious hot spot was developed at the excitation point because of its distance from the reflections. Despite not being able to observe the hot spot in the damaged region directly, the more localized thermal pattern in the central region of the plate, as shown in Fig. 6 (c) and Fig. 6 (d), may be a hint of the heat generated by the friction in the damaged region. Similar to the first test, it again took a considerable amount of time for the hot spot at the excitation point to disappear fully because of the significant amount of energy accumulation due to heat transfer.
Table 4 Compression results, the second vibrothermographic test

\begin{tabular}{lllll}
\hline & $\mathrm{C} 0$ & $\mathrm{C} 1$ & $\mathrm{C} 2$ & $\mathrm{C} 3$ \\
\hline Resolution & $640 \times 480$ & & & \\
Number of Frames & 200 & & & \\
Original Size & $181,554 \mathrm{~KB}$ & & 103.75 & 260.48 \\
CR & 13.99 & 34.43 & 52.9556 & 68.5675 \\
MSE & 14.7387 & 32.0018 & $30.8917 \mathrm{~dB}$ & $29.7696 \mathrm{~dB}$ \\
PSNR & $36.4462 \mathrm{~dB}$ & $33.0791 \mathrm{~dB}$ & 0.7159 & 0.6684 \\
SSIM & 0.9093 & 0.8161 & 0.9966 & 0.9956 \\
Avg. COMAC & 0.9991 & 0.9980 & $1750 \mathrm{~KB}(-99.04 \%)$ & $697 \mathrm{~KB}(-99.62 \%)$ \\
Size (Change) & $12,982 \mathrm{~KB}(-92.85 \%)$ & $5273 \mathrm{~KB}(-97.10 \%)$ & & \\
\hline
\end{tabular}




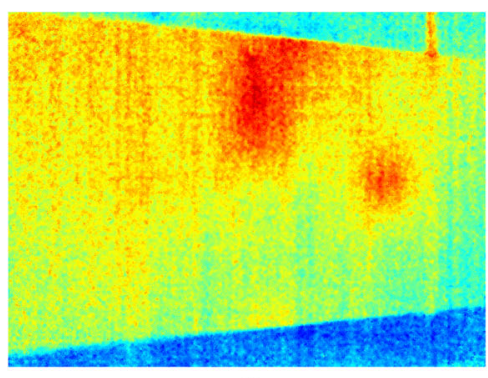

a

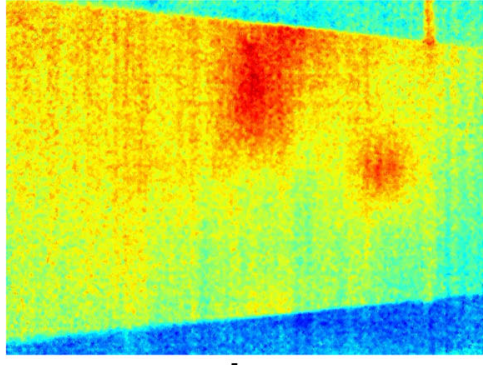

b

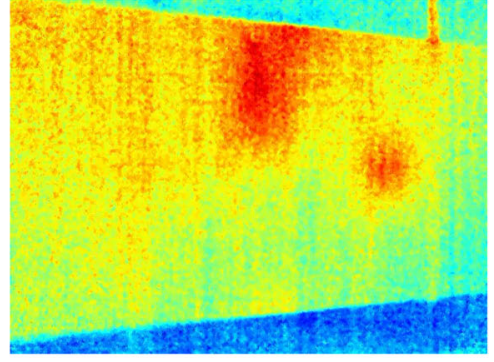

C

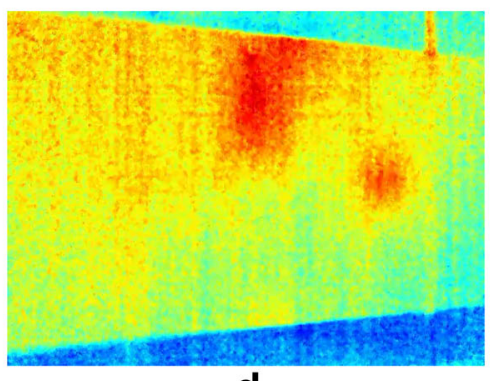

d

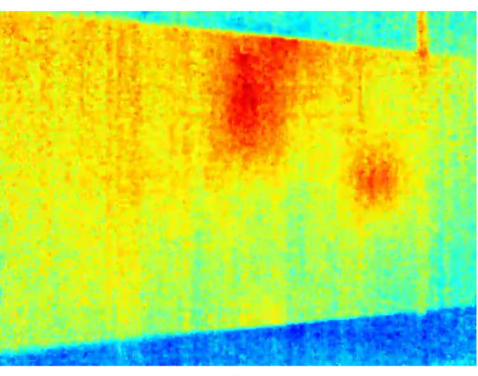

e

Fig. 7 The (a) original, (b) C0, (c) C1, (d) C2 and (e) C3 versions of Fig. 5 (b)

The data recorded during the vibrothermographic tests have confirmed that the friction in the damaged region was able to cause detectable local heat generation. Apart from the main feature, other relatively minor features were also developed and captured in the images, such as the irrelevant hot spots, color fluctuations and reflections, which would add more challenges to the compression process.

For the next step, these images were compressed into four different levels. The CR, MSE, PSNR, SSIM and COMAC were then utilized to compare the original and the compressed images. Next, feature extraction through FastICA was applied to investigate the differences between the extracted components and thus to study the effects of the compression process on the characteristics of each important feature.

\section{Data Processing}

\section{Image Compression}

The data were compressed into four levels (named as $\mathrm{C} 0, \mathrm{C} 1$, $\mathrm{C} 2$ and $\mathrm{C} 3$ ) using the adaptive compression algorithm

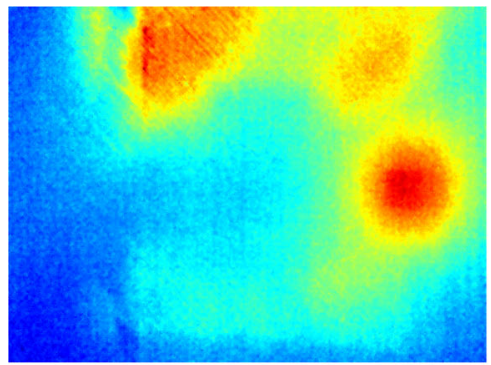

a

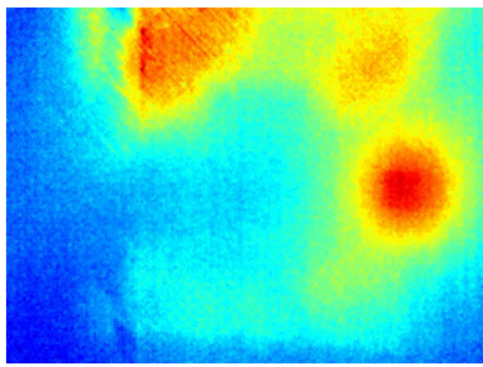

b

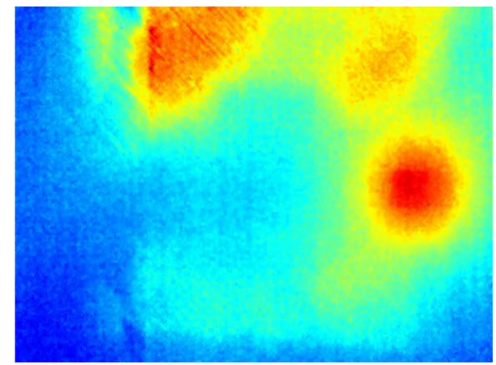

C

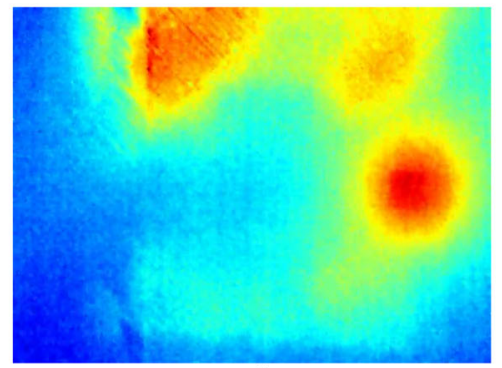

d

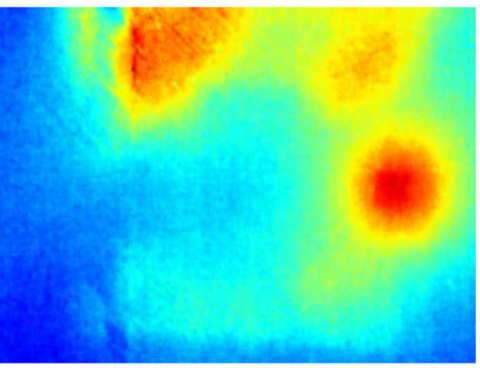

e

Fig. 8 The (a) original, (b) C0, (c) C1, (d) C2 and (e) C3 versions of Fig. 6 (c) 


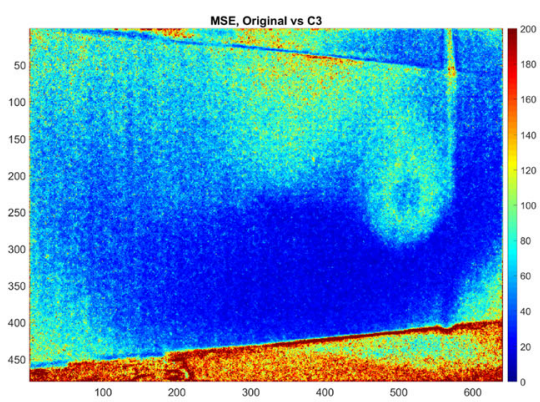

a

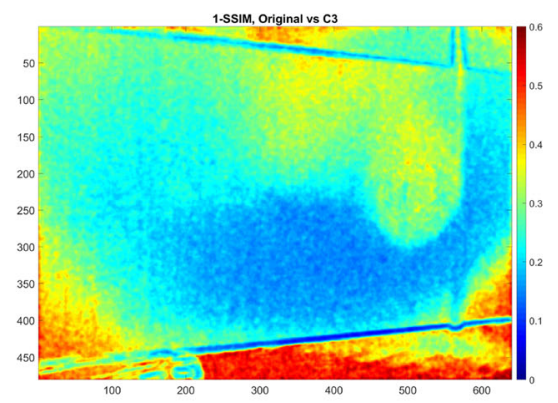

b

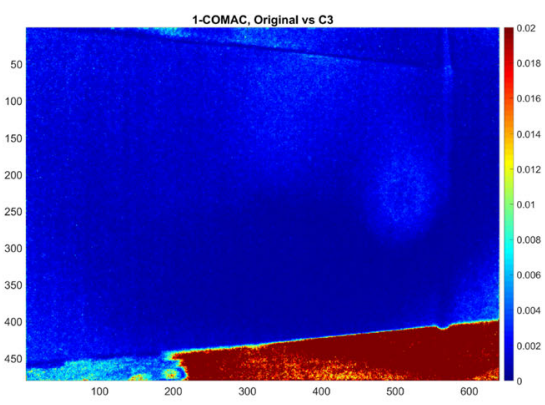

C

Fig. 9 The (a) MSE, (b) (1-SSIM) and (c) (1-COMAC) maps between the original data set and the compressed data set C3, the first vibrothermographic test

described in section "Algorithm overview and theoretical background", where the data set $\mathrm{C} 0$ had the lowest compression ratio while $\mathrm{C} 3$ had the highest compression ratio among these four sets of compressed images.

After the compression process, the methods introduced in the section "Algorithm overview and theoretical background" were applied to evaluate the performance of the image compression process. The results between the original data set and each compressed data set in addition to the basic information of the data and the exact space required for storage of each data set are summarized in Tables 3 and 4. The compressed versions of Figs. 5 (b) and 6 (c) are shown in Fig. 7 and Fig. 8 respectively as examples, in which new colormaps were created and applied based on the jet color scheme in MATLAB.

The values in Tables 3 and 4 demonstrate that the results from all five methods, namely CR, MSE, PSNR, SSIM and COMAC, changed monotonically as the level of compression increased. To be specific, the CR and MSE increased for data with a higher level of compression while the PSNR, SSIM and COMAC all decreased. However, the exact values and the relative changes calculated from each method are not comparable because of the different calculation algorithms of the methods.

By observing the images in Figs. 7 and 8 directly, the characteristics of the major features were relatively unchanged after the image compression. Although it was noticeable that the images had been compressed, the significance of the image compression in different regions of the images was unclear. In order to determine the effects of the image compression process in different regions of the images, the maps of MSE, SSIM and COMAC, which have been explained in the section "Algorithm overview and theoretical background", were calculated between the original data set the data set with the highest compression ratio (C3) and displayed in Figs. 9 and 10. Specifically, the (1-SSIM) and (1-COMAC) maps are displayed so that the regions affected more significantly during the image compression process will appear in red in the generated images.

By observing the images in Figs. 9 and 10, although the maps from the three different methods have dissimilar values and scales because of their different calculation algorithms, it is ascertainable that the regions with greater differences after the image compression process were mostly the areas that did not contain meaningful features, such as the background regions in Fig. 9 and the boundary regions in Fig. 10.

This observation has corroborated the possibility of applying image compression to results from vibrothermography. In vibrothermography or most IRT approaches in general, the hot spots in the damaged regions are likely to change more drastically over time while the irrelevant features in the background will stay relatively unchanged. The high level of activity of the hot spots generated by the meaningful sources means they will remain relatively unaffected during the image
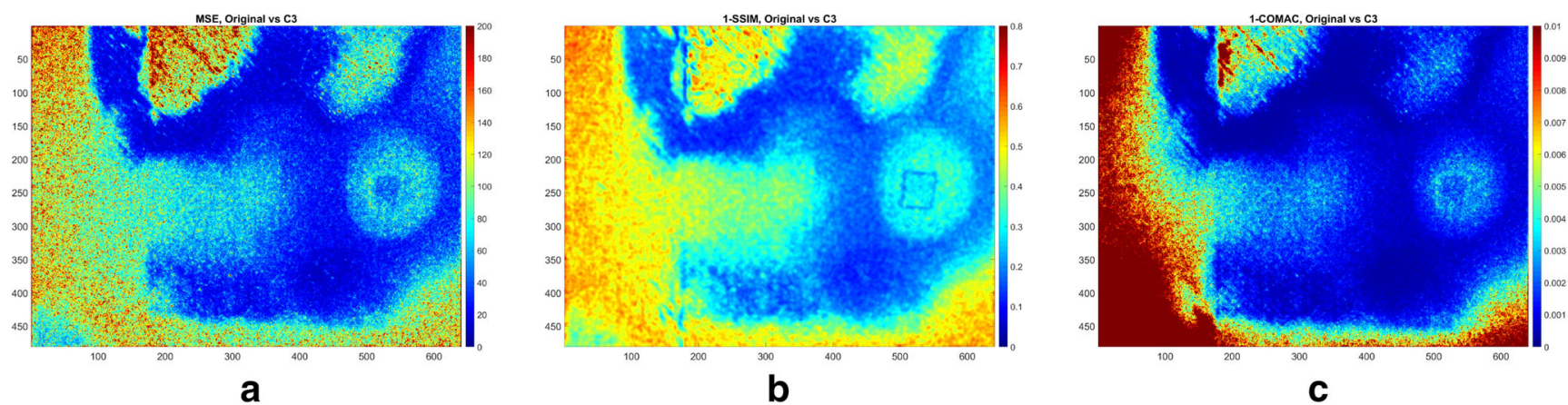

Fig. 10 The (a) MSE, (b) (1-SSIM) and (c) (1-COMAC) maps between the original data set and the compressed data set C3, the second vibrothermographic test 


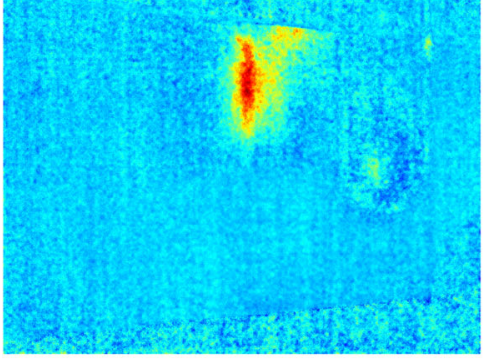

a

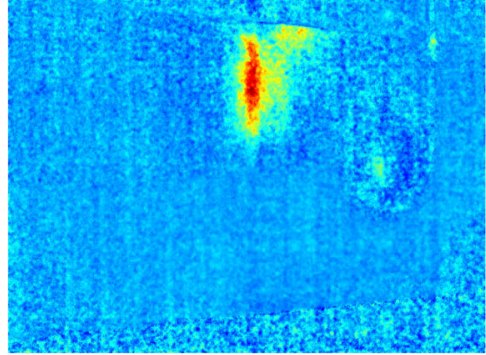

b

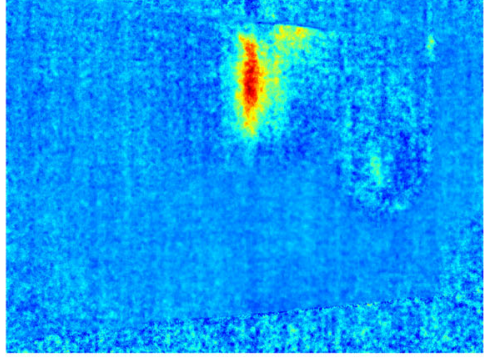

C

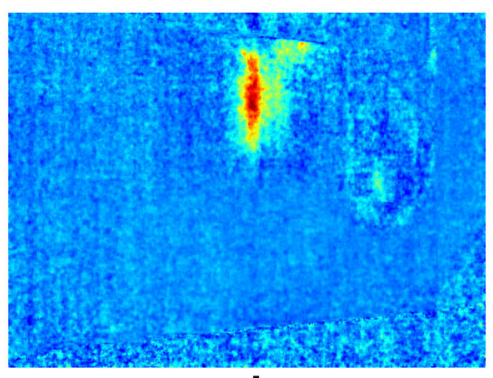

d

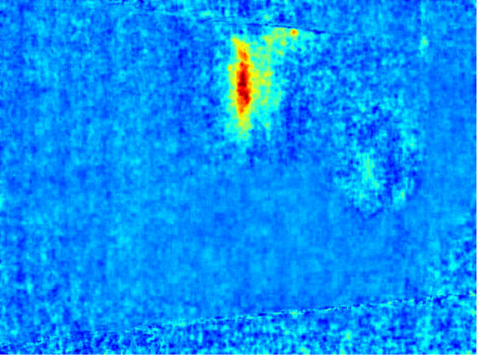

e

Fig. 11 The (a) original, (b) C0, (c) C1, (d) C2 and (e) C3 versions of the extracted hot spot in the damaged region, the first vibrothermographic test

compression process. Conversely, as the features developed by the irrelevant background sources and the reflections are usually static during the measurement, these regions will be affected more significantly during image compression.

Based on the observations described above, it is reasonable to assert that the meaningful features would still be successfully extracted after feature extraction even though the image compression had been performed, and the characteristics of the features would remain mostly unchanged.

\section{Feature Extraction}

After acquiring the four sets of compressed versions of the results from each vibrothermographic test, feature extraction was performed on all ten sets of data using the FastICA described in section "Algorithm overview and theoretical background". Due to the nature of FastICA that has been explained, the number of extracted components was equal to the number of signals provided, which means that there was a large number of extracted components that did not contain

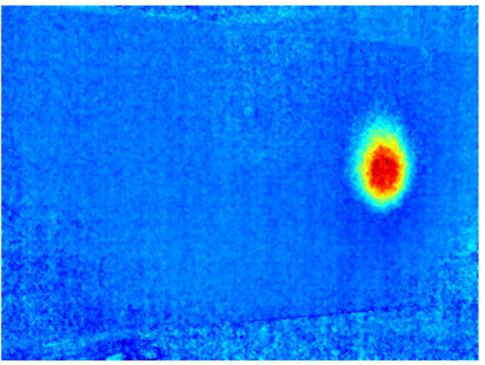

a

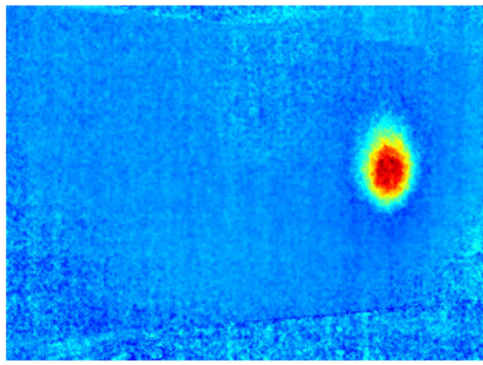

b

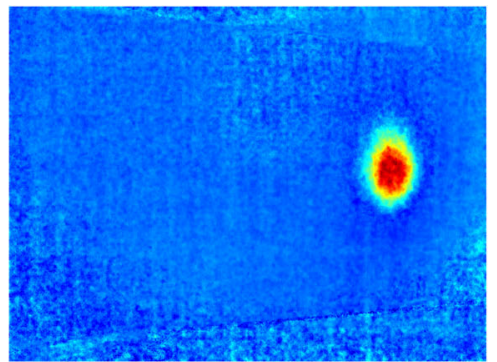

C

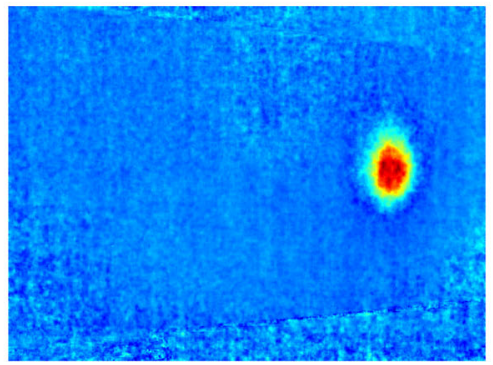

d

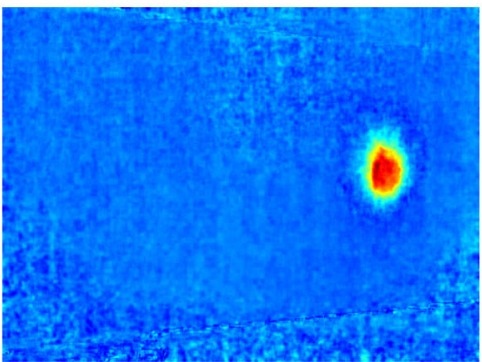

e

Fig. 12 The (a) original, (b) C0, (c) C1, (d) C2 and (e) C3 versions of the extracted hot spot at the excitation point, the first vibrothermographic test 
Table 5 The number of elements (pixels) that each feature consists of, the first vibrothermographic test

\begin{tabular}{|c|c|c|c|c|c|}
\hline & Original & $\begin{array}{l}\mathrm{C} 0 \\
\mathrm{CR}=14.13\end{array}$ & $\begin{array}{l}\mathrm{C} 1 \\
\mathrm{CR}=30.55\end{array}$ & $\begin{array}{l}\mathrm{C} 2 \\
\mathrm{CR}=76.00\end{array}$ & $\begin{array}{l}\mathrm{C} 3 \\
\mathrm{CR}=184.41\end{array}$ \\
\hline Damage & 6730 & 6401 & 6124 & 5808 & 5263 \\
\hline (Change) & $(0)$ & $(-4.89 \%)$ & $(-9.00 \%)$ & $(-13.70 \%)$ & $(-21.80 \%)$ \\
\hline Excitation & 5018 & 4707 & 4549 & 4102 & 3768 \\
\hline (Change) & (0) & $(-6.20 \%)$ & $(-9.35 \%)$ & $(-18.25 \%)$ & $(-24.91 \%)$ \\
\hline
\end{tabular}

Fig. 13 The (a) original, (b) C0, (c) $\mathrm{C} 1,(\mathbf{d}) \mathrm{C} 2$ and (e) $\mathrm{C} 3$ versions of the reconstructed hot spot in the damaged region, the first vibrothermographic test
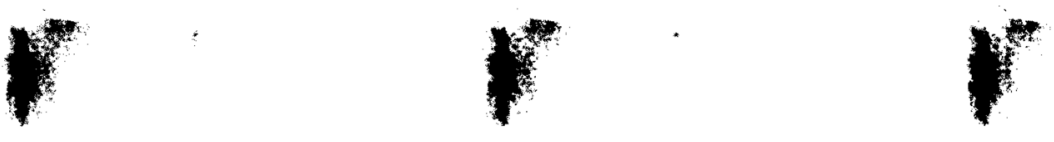

a

C

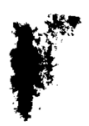

d

e
Fig. 14 The (a) original, (b) C0, (c) $\mathrm{C} 1$, (d) $\mathrm{C} 2$ and (e) $\mathrm{C} 3$ versions of the reconstructed hot spot at the excitation point, the first vibrothermographic test a

C 
any actual meaningful feature. For this reason, the extracted components were reviewed manually, after which two meaningful components in each data set were selected, so that the effects of the image compression process on each meaningful feature could be assessed independently.

In the first data set, the two meaningful features were the hot spots generated in the damaged region and at the excitation point, which are shown in Figs. 11 and 12 respectively.

It is demonstrated in Figs. 11 and 12 that the two important features were extracted successfully despite image compression being performed. The differences between the results from the uncompressed and compressed images are only distinguishable under close inspection. Specifically, the compressed data sets were still able to provide features with correct shape and location. However, the image compression process had marginally influenced the size of the features. By observing closely, the features extracted from images sets with higher compression ratio tended to have a smaller size, which was true for both features.

To explain this, the edge regions of the hot spot always experience less drastic changes over time during the vibrothermographic tests. This means the edge regions were likely to be compressed more significantly, and the size of the area being affected was determined by the level of compression. In this scenario, when the compression ratio increased, a larger percentage of the hot spot would be affected, which correspondingly reduced the size of the remaining feature.

In order to quantify the effects of image compression on the size of the hot spots, each extracted image was normalized to have zero mean and unit variance. If the intensity values of pixels were considered as a probability distribution, pixels constituting the hot spots must lie in the upper-end region of the distribution.
For this reason, it was assumed that elements with values greater than 3 were parts of the hot spots, where the value - 3 - used here was determined after calibrations on this specific data set. The number of elements (pixels) matching this requirement in each extracted component is summarized in Table 5. Pixels not matching this requirement were filtered out. The reconstructed images after the filtering process are shown in Figs. 13 and 14.

Based on the results in Table 5, Figs. 13 and 14, it can be verified that the image compression has altered the size of the features while the shape and location of the feature remained mostly unchanged. The reduction in the size of the features was positively correlated with the compression ratio. The changes on the size of the meaningful features were relatively insignificant considering the high compression ratios, as it has been confirmed through the results from MSE, SSIM and COMAC that the compression process mostly affected the less important areas containing irrelevant features. Specifically, for a compression that could save $97 \%$ of storage space, the reduction of the size of the meaningful features was only less than $10 \%$.

In the second data set, apart from the hot spots in the damaged region and at the excitation point, there were additional features which were the reflections on the plate due to removal of the barrier.

FastICA was still able to separate the hot spots in the damaged region (Fig. 15) and at the excitation point (Fig. 16) from the irrelevant thermal patterns successfully, even though the reflections covered a large portion of the images. However, the thermal pattern of the reflections on the reflective tape was so significant that an independent component containing only this feature was extracted, which caused this area to be removed from the feature of the excitation point, as shown in Fig. 16.

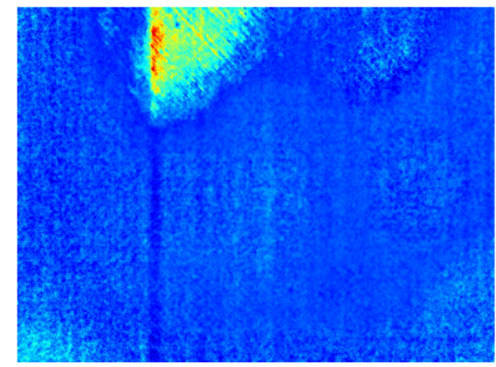

a

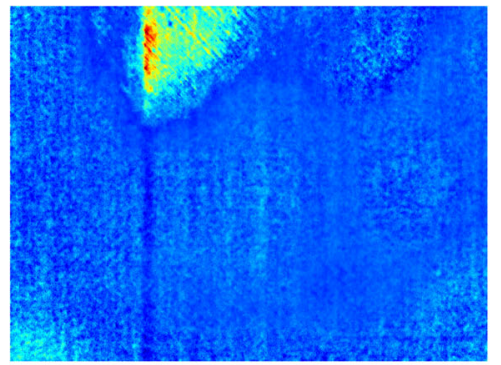

b

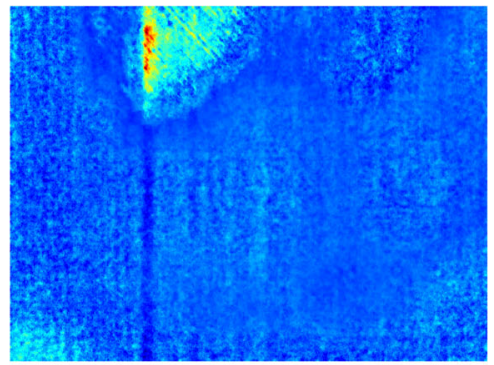

C

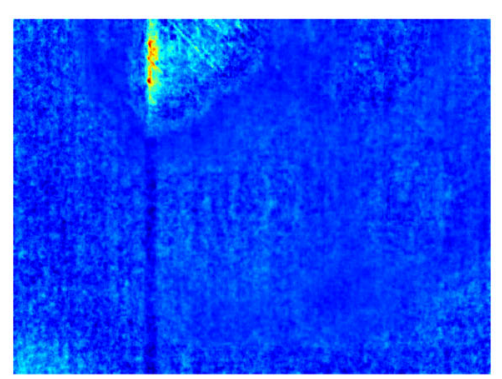

d

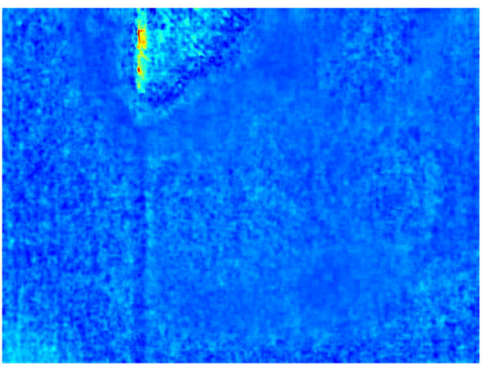

e

Fig. 15 The (a) original, (b) C0, (c) C1, (d) C2 and (e) C3 versions of the extracted hot spot in the damaged region, the second vibrothermographic test 


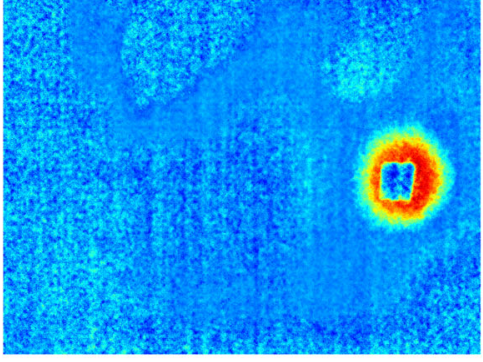

a

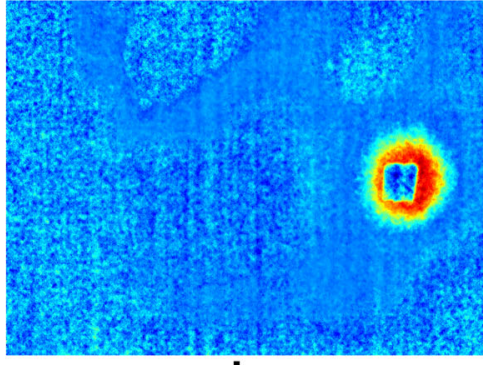

b

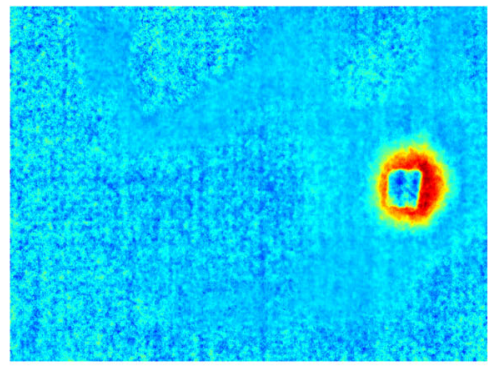

C

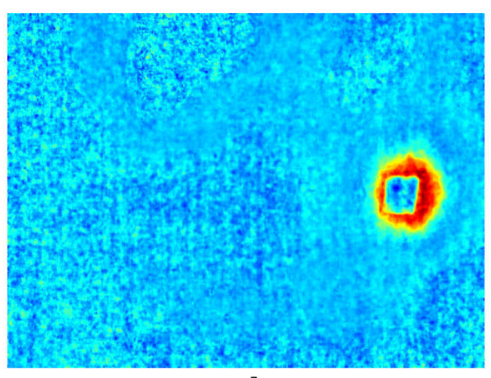

d

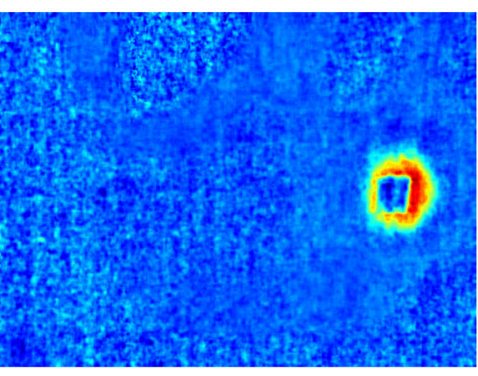

e

Fig. 16 The (a) original, (b) $\mathrm{C} 0,(\mathbf{c}) \mathrm{C} 1,(\mathbf{d}) \mathrm{C} 2$ and (e) $\mathrm{C} 3$ versions of the extracted hot spot at the excitation point, the second vibrothermographic test

By observing the extracted features, it can be inferred that the observations from the first data set still apply and so the same conclusions can still be made. The compressed data were still able to provide correctly identified features. The size of the features decreased more significantly as the compression ratio increased, which has been verified in Table 6, Figs. 17 and 18 using the same technique applied to the results from the first test.

Additionally, in this data set, the hot spot caused by the frictional heat generation in the damaged region was partially mixed with the reflections, as shown in Fig. 15. The image compression process had moderately affected the regions containing the reflections, as shown in Fig. 10, which caused significantly more obvious differences between Fig. 15 (a) and Fig. 15 (b), Fig. 15 (c), Fig. 15 (d) and Fig. 15 (e) compared to the cases in other extracted components. This behavior is clearly reflected in Table 6 and Fig. 17 as the percentage decreases in the number of elements (pixels) from the original set to $\mathrm{C} 0, \mathrm{C} 1, \mathrm{C} 2$ and $\mathrm{C} 3$ are significantly higher compared to the values in the other three features. For this feature, the number of elements (pixels) was reduced by $66.81 \%$ from the original set to $\mathrm{C} 3$ while this number was only $-21.80 \%,-24.91 \%$ and $-23.81 \%$ for the other three features respectively.

\section{Discussion and Conclusion}

In this article, research outputs focusing on the verification of the viability of applying image compression to results from vibrothermographic tests is presented.

It has been shown through results from two tests that the compressed images were still able to preserve the meaningful features, such as the hot spot in the damaged region, with overall accurate characteristics despite the challenges caused by the irrelevant hot spots, fluctuations and reflections in the images.

By using three different methods, namely MSE, SSIM and COMAC, it has been verified that the image compression process was able to have more significant influences in regions mostly consisting of less-important features that did not change drastically across frames in measurement results while having a considerably smaller impact in regions with important time-variant features. By observing the extracted components from the original and the compressed images, it has been discovered that the compression process reduced the size of the important features slightly by compressing their outer regions. The size of the area being removed was positively correlated with the compression ratio, where a larger

Table 6 The number of elements (pixels) that each feature consists of, the second vibrothermographic test

\begin{tabular}{|c|c|c|c|c|c|}
\hline & Original & $\begin{array}{l}\mathrm{C} 0 \\
\mathrm{CR}=13.99\end{array}$ & $\begin{array}{l}\mathrm{C} 1 \\
\mathrm{CR}=34.43\end{array}$ & $\begin{array}{l}\mathrm{C} 2 \\
\mathrm{CR}=103.75\end{array}$ & $\begin{array}{l}\mathrm{C} 3 \\
\mathrm{CR}=260.48\end{array}$ \\
\hline Damage & 9139 & 7992 & 6270 & 4376 & 3033 \\
\hline (Change) & $(0)$ & $(-12.55 \%)$ & $(-31.39 \%)$ & $(-52.12 \%)$ & $(-66.81 \%)$ \\
\hline Excitation & 7262 & 6490 & 5977 & 5707 & 5533 \\
\hline (Change) & $(0)$ & $(-10.63 \%)$ & $(-17.69 \%)$ & $(-21.41 \%)$ & $(-23.81 \%)$ \\
\hline
\end{tabular}


Fig. 17 The (a) original, (b) $\mathrm{C} 0$, (c) $\mathrm{C} 1$, (d) $\mathrm{C} 2$ and (e) $\mathrm{C} 3$ versions of the reconstructed hot spot in the damaged region, the second vibrothermographic test

a

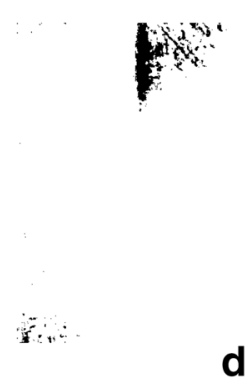

d b

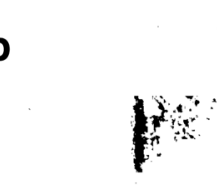

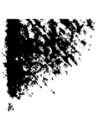

$$
3
$$

C

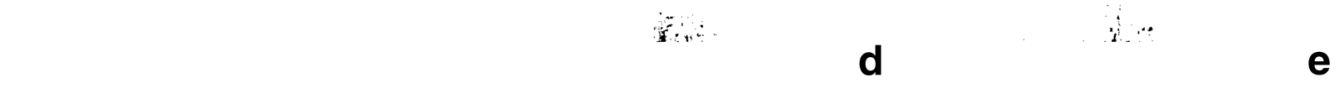

compression ratio always led to hot spots with smaller size. However, the influences on the meaningful features were still significantly smaller than the impact in the less-important regions. Specifically, a compression process which was able to save $97 \%$ of storage space only altered the size of the meaningful features by less than $10 \%$ in this research.

For this reason, a sacrifice of the accurate determination of the size of a feature can be highly beneficial in exchange for the storage space saved from image compression when the requirement on the accurate determination of the size of the feature is not critical. The hot spots extracted from the compressed data are still able to maintain generally accurate characteristics such as the location and shape.

However, it must be noted that in the thermal images used in this research, the regions with valuable information only occupied relatively small areas. For images with larger areas covered

by valuable information, it can be expected that a comparably high compression ratio will not be equally viable because it will potentially lead to a greater reduction in terms of the size of the meaningful features after the compression process.

Aside from the observations and conclusions obtained from this research, there are still potential improvements that can be made.

Firstly, it is possible to use other image compression methods to verify if the same phenomena still occur, which could increase the generality of the conclusions obtained from this research.

Secondly, the structure used in this research is still relatively simple, in which only several features were involved. It can be beneficial to repeat the procedures in this research on a more complicated structure with more complex geometry, which could increase the practicality of this approach.

Fig. 18 The (a) original, (b) $\mathrm{C} 0$, (c) $\mathrm{C} 1$, (d) $\mathrm{C} 2$ and (e) $\mathrm{C} 3$ versions of the reconstructed hot spot at the excitation point, the second vibrothermographic test
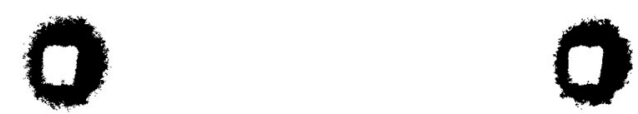

0 
Lastly, although the MSE, SSIM and COMAC were able to provide similar results in terms of the influences from the image compression process in different regions of the images, minor disagreements could still be observed, such as the bottom-left region in both Figs. 9 and 10. The differences have been attributed to the dissimilar calculation algorithm of each method in this article, but it might be worthwhile to look for more specific reasons.

Research Data Accessibility The data produced during this research project and used in this article have been uploaded to Google Drive, which can be accessed at https://drive.google. com/drive/folders/1jEwtYENME9P9qh4isZeS2dTQGZyBvl-n? usp=sharing.

\section{Compliance with Ethical Standards}

Conflict of Interest The authors declare that they have no conflict of interest.

Open Access This article is licensed under a Creative Commons Attribution 4.0 International License, which permits use, sharing, adaptation, distribution and reproduction in any medium or format, as long as you give appropriate credit to the original author(s) and the source, provide a link to the Creative Commons licence, and indicate if changes were made. The images or other third party material in this article are included in the article's Creative Commons licence, unless indicated otherwise in a credit line to the material. If material is not included in the article's Creative Commons licence and your intended use is not permitted by statutory regulation or exceeds the permitted use, you will need to obtain permission directly from the copyright holder. To view a copy of this licence, visit http://creativecommons.org/licenses/by/4.0/.

\section{References}

1. T. Stepinski, T. Uhl and W. Staszewski(2013). Staszewski, Advanced Structural Damage Detection: From Theory to Engineering Applications, Wiley

2. Marinetti S, Grinzato E, Bison PG, Bozzi E, Chimenti M, Pieri G, Salvetti O (2004) Statistical analysis of IR thermographic sequences by PCA. Infrared Phys Technol 46:85-91

3. X Chi, D Di Maio and NAJ Lieven (2019). "Modal-based vibrothermography using feature extraction with application to composite materials," Structural Health Monitoring. [Online First]: https://doi.org/10.1177/1475921719872415

4. J Shlens (2014). "A tutorial on principal component analysis," Int. J. Remote Sens. , vol. 51 , no. 2

5. Lugin S, Netzelmann U (2005) An effective compression algorithm for pulsed thermography data. NDT \& E International 38(6):485490

6. G Schaefer, R Starosolski and SY Zhu (2005). "An evaluation of lossless compression algorithms for medical infrared images," in IEEE Engineering in Medicine and Biology 27th Annual Conference, Shanghai

7. Renshaw JB, Chen JC, Holland SD, Thompson RB (2011) The sources of heat generation in vibrothermography. NDT \& E International 44(8):736-739
8. Henneke EG II, Reifsnider KL, Stinchcomb WW (1986) Vibrothermography: investigation, development, and application of a new nondestructive evaluation technique. Virginia Polytechnic Institute and State University, Blacksburg

9. Zhang Y, Hutchinson P, Lieven NAJ, Nunez-Yanez J (2019) Adaptive event-triggered anomaly detection in compressed vibration data. Mech Syst Signal Process 122:480-501

10. Y Zhang, P Hutchinson, NAJ Lieven and J Nunez-Yanez (2017). "Optimal compression of vibration data with lifting wavelet transform and context-based arithmetic coding," in European Signal Processing Conference (EUSIPCO)

11. WB Pennebaker and JL Mitchell (1993). JPEG: still image data compression standard, Springer US

12. Skodras A, Christopoulos C, Ebrahimi T (2001) The JPEG 2000 still image compression standard. IEEE Signal Process Mag 18(5): $36-58$

13. GJ Sullivan, JR Ohm, WJ Han and T Wiegand (2012). "Overview of the high efficiency video coding (HEVC) standard," IEEE Transactions on Circuits and Systems for Video Technology, vol. 22 , no. 12

14. D Taubman and M Marcellin (2012). JPEG2000 image compression fundamentals, Standards and Practice: Image Compression Fundamentals, Standards and Practice, Springer US

15. Wang Z, Bovik A, Sheikh H, Simoncelli E (2004) Image quality assessment: from error visibility to structural similarity. IEEE Trans Image Process 13(4):600-612

16. NAJ Lieven and DJ Ewins (1988), "Spatial correlation of mode shapes, the coordinate modal assurance criterion (COMAC)," in Proceedings of the Sixth International Modal Analysis Conference, Orlando, FL

17. JV Stone (2004). Independent component analysis: a tutorial introduction, MIT Press

18. A Hyvarinen (1999). "Fast and robust fixed-point algorithms for independent component analysis," IEEE Transactions on Neural Networks, vol. 10

19. A Hyvarinen (2013). "Independent component analysis: recent advances," Philosophical Transactions of the Royal Society

20. Hexcel (2016). "HexPly® 8552 Epoxy Matrix Product Data Sheet,

21. Meola C, Boccardi S, Carlomagno GM (2015) Infrared thermography in the evaluation of aerospace composite materials. Woodhead Publishing

22. Toubal L, Karama M, Lorrain B (2006) Damage evolution and infrared thermography in woven composite laminates under fatigue loading. Int J Fatigue 28(12):1867-1872

23. Bagavathiappan S, Lahiri BB, Saravanan T, Philip J, Jayakumar T (2013) Infrared thermography for condition monitoring - a review. Infrared Phys Technol 60:35-55

24. Avdelidis NP, Hawtin BC, Almond DP (2003) Transient thermography in the assessment of defects of aircraft composites. NDT \& E International 36(6):433-439

25. Goidescu C, Welemane H, Garnier C, Fazzini M, Brault R, Péronnet E, Mistou S (2013) Damage investigation in CFRP composites using full-field measurement techniques: combination of digital image stereo-correlation, infrared thermography and X-ray tomography. Compos Part B 48:95-105

26. Meola C, Boccardi S, Carlomagno GM, Boffa ND, Monaco E, Ricci F (2015) Nondestructive evaluation of carbon fibre reinforced composites with infrared thermography and ultrasonics. Compos Struct 134:845-853

27. DJ Ewins (1984). Modal testing: theory and practice, WileyBlackwell

Publisher's Note Springer Nature remains neutral with regard to jurisdictional claims in published maps and institutional affiliations. 